SIV grown in human cell lines. Similar results were reported by D. P. Bolognesi et al. (IV Int. Conf. Adv. AIDS Vaccine Dev., Marco Island, 1991) who, in addition, found no anti-cell antibodies in monkeys vaccinated with recombinant vectors or subunit vaccines. Second, we absorbed out a significant portion of the anti-cell antibodies by incubating plasmas with uninfected $\mathrm{CEM} \times 174$ cells. Removal of the anti-cell antibodies reduced the cell clumping and growth inhibition, but did not affect the in vitro SIV neutralizing titre. Third, we examined plasmas from the SIV vaccinees for the ability to block binding of monoclonal antibodies (OKT4a and OKB7) to the surface of $\mathrm{CEM} \times 174$ cells, as measured by flow cytometry after live-cell fluorescence staining. Our findings indicated that the anti-cell antibodies present in the plasmas did not interfere with the ability of OKT4a to bind to $\mathrm{CD} 4$, or of OKB7 to bind to CR2 (complement receptor type 2). Finally, we tested the ability of the anti-cell antibodies to block live SIV from binding to CD4-bearing human $\mathrm{T}$ cells and found that SIV binding was not inhibited. Thus, these data suggest that the anti-cell antibodies elicited in response to our whole SIV vaccine preparation do not appear to play a major role in the in vitro neutralization of SIV. Furthermore, it appears that the anti-cell antibodies do not block access of SIV to the surface of target cells.

We should like to mention some other points. Exposure of macaques to the human CD4 protein elicits an antibody response to the human molecule that is capable of blocking SIV infection ${ }^{5}$. Thus, in macaques immunized with uninfected human $\mathrm{CD} 4^{+}$cells, a similar antibody response and effect might explain the observations of Stott et al. In terms of protection conferred by immunization with infected cells, we should note that CD4 expression in infected cells is markedly reduced when compared to uninfected cells. Therefore, it seems likely that specific immune responses to SIV antigens are important in the protection against challenge infection. Of course, we cannot exclude a combination of both mechanisms. Additional evidence for the role of an SIV-specific immune response in protection is found in the work of Putkonen et al. ${ }^{7}$, who showed that passive transfer of pooled serum from experimentally infected macaques to naive macaques provided protection from challenge infection. Because we did not find anti-cell antibodies in plasmas from our experimentally infected macaques, we assume that none were present in the macaques of Putkonen et al. ${ }^{7}$. Taken together, these data suggest that SIV-specific immune responses do provide protection, but that additional means (for example, CD4 immunization) of inducing protection should be considered in an overall vaccine strategy for AIDS.

Department of Pathology,

DAVID C. MONTEFIORI

Vanderbilt University Medical School, Nashville, Tennessee 37232, USA

VANESSA M. HIRSCH

Laboratory of Infectious Diseases,

NIAID/NIH, Rockville,

Maryland 20852, USA

Department of Pediatrics,

PHILIP R. JOHNSON

The Ohio State University,

Columbus, Ohio 43205, USA

SIR - Maddox's otherwise helpful article $^{1}$ began and ended unfortunately. I fear that the structure of its assessment of Hoffmann's and Stott's work is going to yield all sorts of bizarre press reports, leading to confusion among the general public.

As I understand it, Duesberg essentially finds the lack of fit of HIV into Koch's and Rivers' postulates as grounds to dismiss HIV as significant in the aetiology of AIDS. Indeed, HIV doesn't fit these guidelines, and the more that is learned about it the more evident it becomes that just as Koch's postulates had to be modified by Rivers to account for the differences between bacterial and viral pathogenesis, so it may be necessary to devise new postulates to describe the relationship between lentiviruses, even all retroviruses, and their hosts.

Basically, Duesberg's objection is that the pattern of HIV-host interaction is

\title{
Efficiency in a liquid solar cell
}

SIR - We have demonstrated ${ }^{1}$ the effect of cyanide on CdSe ferrocyanide photoelectrochemical cells (PECs), resulting in the highest solar-to-electrical conversion efficiency for a PEC. It has been suggested that $26 \%$ of the observed photocurrent was due to a "virtual battery" resulting from electrochemical discharge of cyanide to cyanate at the photoelectrode ${ }^{2}$. We do not observe this battery effect, and our investigations indicate that results in ref. 2 supporting the effects are artefacts of the experimental configuration in that study.

Specifically, in a conventional PEC, without storage $^{3}$, the electrochemical reactions occur within a single cell. However, the "battery effect" study used a separate anode/cathode compartment cell $^{2}$ which artificially concentrates ferricyanide in the cell. This exaggerated ferricyanide chemically reacts with cyanide creating the reported cyanate buildup. Our further experiments indicate that no cyanide is electrochemically oxidized at either $\mathrm{CdSe}$ or at $\mathrm{Pt}$ electrodes. A simple non-electrolysis dark not yet clearly delineated, and that no obvious, standard, known pathobiological mechanism easily succeeds in establishing HIV as the aetiological agent of AIDS. Just as autoimmunity figures in many human diseases, such as the production of cross-reactive antibodies in response to $\beta$-haemolytic streptocci which subsequently mediate rheumatic carditis, so may the reports by Kion and Hoffmann ${ }^{8}$, and by Stott et al. ${ }^{3}$, lead to a picture of AIDS pathology involving an autoimmune process triggered by HIV. Novel aspects of immune function may well be part of HIV-mediated pathology ${ }^{9}$, and viral latency further complicates fitting HIV into standard pathobiological models ${ }^{10}$.

Maddox wrote as much in his article, yet the opening and closing sentences are what the popular press will notice. This causes confusion for all concerned: false and misleading hopes for those directly engaged with the disease, and weeds to be uprooted by those of us involved in education.

ANDREW P. WHIPPLE

Biology Department, Taylor University, 500 Reade Avenue, Upland,

Indiana 46989, USA

1. Maddox, J. Nature 353, 297 (1991).

2. Anderson, C. Nature 353, 287 (1991)

3. Stott, E.J. et al. Lancet 336, 1538 (1990)

4. Stott, E.J. et al. Nature 353, 393 (1991).

5. Watanabe, M. et al. Proc. natn. Acad. Sci. 88, 4616 (1991).

6. Johnson, P. R. et al. Proc. natn. Acad. Sci. (in the press).

7. Putkonen, P. et al. Nature 352, 436-438 (1991).

8. Kion, T. A. \& Hoffmann, G. W. Science 253, 1138 (1991).

9. Imberti, L. et al. Science 254, 860 (1991).

10. Garcia-Blanco, M. A. \& Cullen, B. R. Science 254, 815 (1991). cell shows that all cyanate is due to chemical and not electrochemical origin and hence is not related to photocurrents. The rate at which cyanide and ferricyanide react is controlled and minimized by solution modification techniques ${ }^{4}$, which optimize speciation in solution. Finally, our repeated studies show identical photocurrents for a properly prepared $\mathrm{CdSe}$ ferro/ferricyanide cell both with and without added cyanide. The contention of different quantum efficiencies without cyanide ${ }^{2}$ is not reproduced. Interested readers can find expanded details of this correspondence in ref. 5 .

STUART LICHT DhARMASENA PERAMUNAGE

Department of Chemistry,

Clark University, Worcester,

Massachusetts 01610, USA

1. Licht, S. \& Peramunage, D. Nature 345, 330-333 (1990).

2. Seshardi, G., Chun, J. K. M. \& Bocarsly, A. B. Nature 352, 508-510 (1991).

3. Licht, S., Hodes, G., Tenne, R. \& Manassen, J. Nature 326, 863-864 (1987).

4. Licht, S. Nature 330, 148-151 (1987)

5. Licht, S. J. electrochem. Soc. (in the press) 